\title{
Methodological Approach of Integrating Voronoi Diagram into Space Syntax
}

\author{
[ Ali Essam El Shazly ]
}

\begin{abstract}
Common property of convex space in Voronoi Diagram and Space Syntax prospects their hybrid merge into one interdisciplinary method of cognitive structure. The theoretical review observes both methods under the same condition of spatial convexity with different spatial structure. The essence of space syntax develops its origin of invisible spatial logic into visibility and architectonic conceptions of virtual reality. On the other, the voronoi diagram determines the spatiotemporal automation of all generative space. Supposing the swap of either 'convex hull' shifts the cognitive structure of space into a new empirical realm of 'voronoi-syntax' through their merged tool-box of spatial analyses. Thus, apply universally on multidimensional digits of future space.
\end{abstract}

Keywords - voronoi diagram, space syntax, convex hull

\section{Introduction}

The research advancements in both professions of voronoi diagram and space syntax explore common interest in the cognitive structure of space. In the voronoi process, the space is conceived through the automated partitioning of database structure and geometric modeling, with perceptual dimensions of humanity as well [2]. Meanwhile, space syntax determines the social logic of space according to the largest subdivision of space in the $x-y$ planar coordinates with graph representation [10], which has been questioned for its digital limitation when processing the datasets [11]. This study attempts to funnel the two thoughtful methods of voronoi diagram and space syntax in one complementary field of study. The spatial objective is to exploit the comprehensive tools of cognitive stances that both professions offer, though in separate. Nonetheless, the output may adjust to a wide range of scientific concerns, and not only the cognitive structure of built studies. In search for a common theme to amalgamate the voronoi diagram with space syntax, and through the review of conceptual background behind each, the issue of spatial partitioning is found to be the sieve of building-up the layers of spatial data. In particular, the conceptual unit of convex space is a common vocabulary among both literatures awaiting observation. Convex space itself, however, is a standalone property of space that has no precedence or innovation by either studies. Thus, the matching question of; "which convex space applies for which cognitive structure?" is the key issue to think of for perceptive and conceptive configuration of space. On resolving this pivotal inquiry, numerous spatial layers of vector and/or raster image with tabular database would be managed and analyzed. Through this interfacing framework of convex space, the digital cognitive structure of built (or unbuilt) space shall have methodological reconsideration in future universal scopes.

Ali El Shazly, Associate Professor

Department of Architectural Engineering / Fayoum University Egypt

\section{Methodological Approach}

The spatial interdisciplinary cognitive studies of 'Voronoi Diagram' and 'Space Syntax' demonstrate discursive analytical methodologies of spatial tessellation. On one hand, conceptual application of voronoi diagrams tessellates the space into weighable n-dimensional convex hull, which is analyzed for scientific operations of universal informatics. The statistical modeling enables voronoi of various spatial processes such as assignment, growth, spatiotemporal and two-species models. Also the point pattern analysis covers the numerically based methods of polygon, triangular, nearest neighbor distance, shape of point pattern, spatial intensity, segmenting point patterns, in addition to modeled point processes. The same voronoi conceptions are used for locational optimization of points and lines with their transform overtime. Through the deterministic criteria of voronoi informatics, the field of GIScience ties with other disciplines in spatial problem solving. On the other, the concept of space syntax, which tessellates the open space into broadest two-dimensional convex hull, determines the spatial logic of societies. This logic is derived from converting the convex layout, with its axial representation according to line of sight, into graphs of numerous spatial measurements. The syntactic properties of constructing the convex/axial maps with their graphs determine the indexes of edges and vertices, space-building interface and its converse, decomposition graph and its converse, convex/axial ringiness, connectivity and justified graphs. These visually-based numerical properties allow looking at the spatial system from all points at once with the symmetric/asymmetric depth measure of global spatial integration, and also the distributed/nondistributed measure of local spatial control at layout of urban and building resolutions.

Despite their analytical divergence, both fields of info- and logic-space provide methodological approaches towards the cognitive structure of space through various tools of spatial measurements. Though of different configuration, the essential profile of spatial convex unit in the two methods founds the basis throughout the analytical processing. The common coring property of convex hull supposes the methodological adaptability of cross-applications between space syntax and voronoi diagram in one extended merge. The prospects of convex space among both platforms is investigated in progressing approach from the very basic to the most advanced interconnections. In this emerging study, the essence of mapping the convex hull in space syntax is compared to the tuned-up nonempty voronoi tessellation of explicit convex hull. Meanwhile, the same voronoi convex hull allows the spatial analyses of its own and those merging measurements of space syntax as well. In this respect, the polygonal function of first-order voronoi perceives the nonempty built-up or open 
context of the convex hull, in addition to the nonvisible spatial integration and control measurements of global and local structures in space syntax. Overview of the methodological approach supposes the applicable swap of spatial analyses on either convex hull of voronoi diagram or space syntax without having to reinvent neither wheel, which expands the cognitive structure of space morphology. Future research would stretch the convex hull into higher order 'Voronoi Diagram' and its dual triangulated 'Delaunay Diagram' with their geometrical moments, graph algorithms and statistical explorations in connection to the logical measurements of space syntax.

The current research studies in both fields of spatial information science and space syntax have raised some of their interrelated issues with progressive applications ahead. B. Jiang, and C. Claramunt, reconstructed the non-automatable linear vista of space syntax (the axial-map) into automatable visibility graph structure, which concludes comparable spatial measurements to those counterparts in the classical space syntax accounts, thus allowing the GIS database integration into the urban morphology of space syntax [11]. W. Luo, and A. Mac Eachren, in their effort of knotting the spatial with the social cognitive interaction address the needed design of GIS visualization tools through interdisciplinary state-of-the-art development [59]. Conversely, pure socioeconomic studies themselves capitalize on the GIScientific techniques in unlimited applications, such as the study on poverty using the geo-statistical analyses of spatial mobility in the continual rural migration phenomenon of metropolitan Cairo [25]. Besides, M. Goodchild, recalled the scientific evolution of geographic information in chronological milestones, which in part noticed the gap at present between GIScience and architectural design beyond the 2D emphasis [40]. Having mentioned this, the attempted research encounters this issue from the voronoi standpoint where the nonempty convex hull enables the two-dimensional spatial measurements in relationship to the functional 3D building characteristics, which may also extend to the fourth time dimension by virtue of socioeconomic and building transformations. The encapsulated abilities of convex unit may offer future envision for multidimensional GIScience with the architecture of the cityscape. S. Winter, and K. Freksa, challenged the place in cognitive science as related to the stimuli of anchoring functions in space by contrast to others, regardless of coordination system or centroids of spatial positioning [57]. Interestingly, the metaphor of linguistic syntax or hand sketching demonstrates the mental representation of place in space. Hence the unit of space becomes a discrete cognition of place instead of the continuous auto-geometric spatial tessellation. Of major importance in this argument is the refined spatial boundary into cognitive peripheries containing image of value or identity that can be sensed as place more than being superimposed partitions on space. H. Frichot, traces futuristic diagramming change from real demonstration to hidden imaginative dynamics of spatial conception [24]. Further trajectory methods observe the dynamic spatiotemporal contexts at different scales. This type of study shifts the spatial experience from static capture at instant timing into dynamic data of cross-scale resolutions in time frames [e.g. 4 \& 35]. In this manner, the cognitive advancement would overlay the animated sceneries over the course of time through the various speeds of movement (vehicular, cycling, pedestrian, monorail, subway, aircraft! and so on) and by different people or social stratum (inhabitant, visitor, elderly, child, low-income, robot! ...etc.) in open ended research. From the point of view of this prospected research, the spatial trajectory represents a superstructure of cognitive motion picture, which situates above the foundational layer of convex space.

The other campaign of space syntax attempts to rediscover the social logic of space from various topologic and geometric approaches. Co-authors, B. Hillier, and J. Hanson, advance their cornerstone study of space syntax through the respective studies of retrieving the abstracted social networks into formal geometrical and logical schemes with socio-spatiotemporal dynamics [6], and the spot rays of open 'isovist' visibility dimension in the abstracted logic of spatial decodes [29]. Both studies overcome the nonvisible configuration of classical space syntax with enhanced human cognition of space. B. Hillier, et al. further develops the scale-less space syntax in determinate measure of block-sizing, which results from the topological integrative-weighing of spatial relations at the foreground city-scale street networks, compared to the true geometrical metric-weighing of patchwork areas in the residential background [8]. Hiller's beforehand study entitled 'Space is the Machine' has partitioned the space according to the positional criterion of block-size along the spatial grid, with the layout objective of achieving the 'universal distances' (distances of all spaces to all others) in shortest mean lengths [7]. Parallel result of F. Otto, adapts the logic of grid patterns including the 'Voronoi' norm for network connectivity having the least distances of point distribution against the territorial area [21]. L. Helme, et al.post the notion of logical diagram with the opening statement: "Despite five decades of research into layout automation ........., no automated layout programme is used as standard within the architectural profession." [36, p.34]. Their enthusiastic proposal converts the conceptual bubble diagram into 3D spatial algorithms of most efficient social connections through minimal paths. In mathematical terms, B. Hillier, et al. resolve the paradox of more choice (betweeness) in less integrated graph layout through the parametric inclusion of the vertex degree of connectivity for normalizing the abstract space syntax measurement [9]. Also M. Kruger, and A. Vieira, correspond the standard deviation of spatial dispersion to the mean depth values of relative asymmetry (centrality) in the scaled measure of spatial integration [41]. So far the methodological developers of space syntax represent a regenerative apparatus between in and outdoor dimensions of spatial reality. P. Osmond, regenerates the apparatus of space syntax into convex, isovist and fractal criteria, where the employed convex space acts as the fundamental unit of physical and psychological cognition of urban form [49]. Here the centroids of convex spaces are taken as the origin of generating threedimensional isovist images of conceiving the surrounding surfaces in 360-degree round angle, with their changing skyline and fractal measurement of object deformation at various scales, thus recognizing the visual diversity of space. Although the study directly touches the convex space as the atom of generating space syntax, the developed method is molded in the space syntax norm of spatial tessellation itself, 
which is taken as a matter of fact without reasoning. In another study, M. Ostwald, reversed the space syntax conception to conclude that geometry may have a potential role over topology in shaping spaces [42]. When seen together, Osmond's and Ostwald's studies end from where the voronoi conception takes over; that is the convex hull analyses of geometrically deterministic elements. In a relevant issue of $\mathrm{N}$. Dalton, and R. Dalton, the motional barriers at different heights of isovist visibility affects space syntax [45], which is also found in the case of ordinary voronoi spatial subdivisions. Intuitive treatment freezes the spatial vertices and activates the linking edges at different graph layers of correspondent vision/route adjacency, with aggregated spatial measurements for disaffected logic of space syntax. The voronoi conception itself encounters this barrier issue where the convex hull may consider (if necessary) the unobstructed spatial adjustment. Comparable argument of D. Koch, enriches the architectural theory by recognizing some socio-spatial visible-accessible configurations of captured phenotype space syntax in building design [17].

Apart from the core monitoring of space syntax, some other studies explore the external role of the method. For example, L. Marcus, extends the analytical scope of space syntax in correlation to the urban planning morphology of building/population densities with accessible diversity and movement as applied on part of Stockholm [37]. In the same manner, Y. Ye, and A. Van Nes, compute the matured growth of vital urbanism through the GIS capacity of correlating integral space syntax with building densities and land use diversity [60]. The visually-based isovist software of $\mathrm{H}$. Markhede, et al. develops a spatial positioning tool that can sketch and analyze the overlapping spaces of visibility and integrity in layered interactive graphs [26]. The isovist computation originates from Turner's Depthmap program of visibility graph analysis [5]. S. Psarra, and S. McElhinney, later developed the isovist diagraming into dynamic over-thecorner stochastic modelling [56], in addition to T. Varoudis, and S. Psarra, extension into the isovist 3D realm of space design [58]. Crossover vision of $H$. Schnadelbach, communicates videoing with architectural space in remote 3D topological dynamics of social interaction [27]. In a different computing, S. Hanna, examines the reversed genetic analogy of space syntax from phenotype (product) to genotype (gene) through the artificial intelligence of logically inferred building design [53]. The description of real world (product) defines its representational tools (genes) for creating a new real world. The two-way process, thus, represents the spatial-formal interaction in digital media between space syntax and architecture. Meanwhile, S. Psarra, reclaims the conceptual architectonic form from the space syntax's ghost of spatial configuration, which addresses the missing knowhow of shaping non-discursive into discursive consciousness while making up the whole cognitive theory [55]. It is worth mentioning that the key issue of adjoining convex space may pave the way for a transparency interface between the spatiotemporal perception and the conception of geometrical grid at random. J. Steen, and H. Markhede, stretch-up the argument by showing the incompliance of social interactions at workstations with the measured syntax of integral spaces in selected office buildings [31]. As a result, specific building typologies may raise visual, noise or hierarchal dimensions of physical design over the spatial configuration of deductive circulation patterns. On the contrary, M. Serra, and P. Pinho, capitalize on the scale-less topology of space syntax to adapt at giant spatial systems without loss of generality [43]. Apparently, most of research efforts in space syntax are directed towards the bridging from perceptive spatial configuration into the virtual reality of conceptual design at different levels of building resolutions, with little or no attention paid to the driving 'basic design' force of interchangeable convex space.

Wider scope of cognitive studies defines the built environment from various points of view. To mention some, A. Christopher, et al. codifies any required urban structure according to resolved patterns of grammar-like shaping, which is empirically regarded as the best physical arrangement to be conceived, and from which phenotype space syntax has seen light [1]. In contrary to J. Jacob, however, the tree-like arrangement separates any essence of physical overlaps in urban diversity with the community surveillance over public space, also from which genotype space syntax has seen light [30]. In-between perception of O. Newman, secures the hierarchal semis from public to private space [46]. Meanwhile, K. Al-Sayed, sketches the grammatical shaping of spatial structure, without underlying the mental 'grid-hull' however [32]. E. Llabres, and E. Rico, add time span in the territorial transformation of space as a result of the interactive equilibrium between the natural landscape and the shaped socioeconomic fluidity [20]. The philosophical origin of empiricism dates back in 1620 when F. Balon, denoted the sensual experience of the surrounding world [22]. In this sense the nearer study of G. Cullen, in 1961 experiences the visual townscape in sequential cognitive attractions of space [23]. Counter rationalism refers back to R. Descartes (1597-1650), whom denied the sensual evidence but searching for universal truths of logical thinking [22]. In 2014 J. Burry, constructs the architectural model after Descartes using modern geometrical techniques in analogical space syntax of generative random process [28]. Back in 1782 N. Le Doux, rationalized fortytollgates of Paris in pure geometry. Also the post French revolution since 1848 had inspired Haussmann to plan wide geometrical boulevards throughout historical Paris for security reasons [54]. Most cities of the world followed suit, except C. Sitte, whom in 1889 criticized Haussmann's rigidity with the lost cognitive proportions of space enclosure [16]. The later pragmatism of C. Peircel, et al. reconsidered the practical consequences of conceiving industrial objects [22]. In a more responsive city gardening versus modern industrialization, E. Howard, retreats the breakout of suburbs through independent urban into rural satellites [19]. The idea was based on B. Parker, and R. Unwin, design of vernacular countryside suburbs [13]. Shortly afterwards, R. Unwin, proposed the twelve-per-acre ratio of single-family housing in gardens, which proves to be the ideal socioeconomic realization of human space [51]. Originally, F. Olmsted, developed his perceptual beautification program of American cities according to the park system [14]. Thus, empiricism and rationalism together contradict but complement each other in industrial shaping of modern space. 
The debate of architectural movements since early modernism onwards roots in Louis Sullivan's initiative of 'form follows function' and Mies Van de Rohe's aphorism of 'less is more' that implies the simplified neo-rationalist abstraction [44]. The resulted social divergence from architecture in modern time has caused what can be termed 'rational empiricists' to search reengaging them as is. In this context, urban-scale modernism has been met by vital methods of human factor in cognitive design. C. Rowe, and F. Koetter, propose the collage city of figure-ground massing in the cognitive structure of urbanism [15]. R. Krier, further composes geometric elements to conceive the spatial connection between function, construction and architectonic form of scaled urban fabric [50]. The subtle urban form of S. Kostof, recognizes the spatial grid in shaping parcelled block characteristics throughout history [54]. Basically K. Lynch, classifies the city form into three models of cosmic holiness, practical machinery or bioorganic morphology [33], which in any case is mentally imaged through the skeletal elements of paths, districts, edges, landmarks and nodes [34]. In an antimodernist tone, A. Rossi, defines the urban artefact as the autonomous structure of historical analogue [48]. According to P. Geddes, the culture of city life determines the profound evolution of spatial form [3]. L. Mumford, further empowers the city with concentrated wealth and culture of a community [38]. E. Bacon, conceives the urban culture as the point of contact between mass and space for growing multipath experiences [18]. S. Chapin, et al. rationally assumes the cultured city structure worldwide to be the land use planning of human functions and amenities in geometric models with explicit zoning policies [52]. The furthest 'Conzenian' urban morphology puts it all together in recognizing the interrelated block-plot-building formation of urban tissue [39]. The logical wayfinding in complex urban flows adopts 3D spatial networks of graph theory matrices [47], with the breakout of interdisciplinary geospatial modeling in all structural aspects of urbanity according to the technological GISystems/ Science/ Services/ Studies terms of syntactic compilation [12].

Overview of theoretical cognitive structure dynamically seesaws from empirical to morphological and systematic urban analyses through selective rather than collective criteria. The still unknown mystery of holistic cognitive determination points out the anonymous baseline behind the generative spatial structure. In another words, there should be found deep-water that is deeper than the already invisible configuration of spatial structure so as to recognize the encapsulated urban phenomena. Even though the cognitive structure shares spatial interests between architectural and geographical concerns, the latter holds the containment property of building threshold. GIScience, thus, paves the way for the cognitive structure to integrate with space syntax. In this particular regard, the wide range of multidiscipline cognitive research tackles the analytical techniques of GIScience and space syntax from perceptual, geometrical and topological perspectives, but none of them has yet attempted to insight the very basic structure of the convex space itself with its potential for rebuilding the methodological strategy upon. The common issue of spatial convexity may not only join the cognitive breakdown, but also prosper their analytical methods among each other. The chaining extensions of convex space prospects both conceptual and perceptual dimensions of spatial structure, which is demonstrated in one approach of this study by the adaptable unit of transparent spatial convexity in interrelated applications between the ordered voronoi diagram and space syntax.

\section{Conclusion}

The interactive foundational layer of 'convex hull' with separate configuration in voronoi diagram and space syntax observes their matching of analytical tools in one extendable cognitive structure of space. Despite this fact, the theoretical review in the field of space syntax explores methodological developments towards the conceptual design of virtual space. In this regard, the syntactic innovations are concerned with the spatial visibility issues of architectonic dimensions without arguing the fundamental structure of convex space however. On the other, the inferential approaches of spatial informatics automate the voronoi diagram towards the universal modeling of space in time, without cognitive demonstration of designing the cityscape resolutions however. Meanwhile, the wide range of cognitive studies in architecture and urban planning crosscorrelate the spatial structure issues from various perspectives without a unifying approach however. These divergences of the space syntax from the voronoi diagram with the variety of cognitive studies overview their holistic structure through the framework of convex unit in common digitals of future space.

\section{Acknowledgment}

Gratitude to the Japan Society for the Promotion of Sciences for the scholarship award, which resulted in this research.

\section{References}

[1] A. Christopher, S. Ishikawa, M. Silverstein, M. Jacobson, I. FinksdahalKing, and S. Angel, A Pattern Language: Towns, Buildings, Construction, Oxford University Press, 1977.

[2] A. Okabe, B. Boots, K. Sugihara, S. Chiu, and D. Kendall, Spatial Tessellations: Concepts and Applications of Voronoi Diagrams, Second Edition, Chichester: John Willy \& Sons, 2000.

[3] A. Rossi, The Architecture of the City, Cambridge, Massachusetts: The MIT Press, 1982.

[4] A. Soleymani, J. Cachat, K. Robinson, S. Dodge, A. Kalueff, and R. Weibel, Integrating Cross-scale Analysis in the Spatial and Temporal Domains for Classification of Behavioral Movement, Journal of Spatial Information Science, vol. 8, 2014, 1-25.

[5] A. Turner, Depthmap: A Program to Perform Visibility Graph Analysis, Proceedings of the Third International Space Syntax Symposium, Atlanta, 2001, pp.311-319.

[6] B. Hillier, What do we Need to Add to a Social Network to Get a Society? Answer: Something Like What you have to Add to a Spatial Network to Get a City, The Journal of Space Syntax, vol.1-1, 2010, pp.41-58.

[7] B. Hiller, Space is the Machine: A Configurational Theory of Architecture, Cambridge: Cambridge University Press, 1996.

[8] B. Hillier, A. Turner, T. Yang, and H. Park, Metric and Topo-Geometric Properties of Urban Street Networks, The Journal of Space Syntax, vol. 2-1, 2010, pp.258-279.

[9] B. Hillier, T. Yang and A. Turner, Normalizing Least Angle Choice in Depth Map and How it Opens Up New Perspectives on the Global and Local Analysis of City Space, The Journal of Space Syntax, vol. 3-2, 2012, pp.155-193. 
[10] B. Hillier, and J. Hanson, The Social Logic of Space, Cambridge: Cambridge University Press, 1984.

[11] B. Jiang, and C. Claramunt, Integration of Space Syntax into GIS: New Perspectives for Urban Morphology, Transaction in GIS, vol. 3-6, 2002, pp.295-309.

[12] B. Jiang, and X. Yao, Geospatial Analysis and Modelling of Urban Structure and Dynamics, Springer, 2010.

[13] B. Parker, and R. Unwin, The Art of Building a Home: A Collection of Lectures and Illustrations, London: Longmans, Green, \& Co., 1901.

[14] C. Beveridge, The Papers of Frederick Law Olmsted: Creating Central Park, 1857-1861, Johns Hopkins University Press, vol. III, 1983.

[15] C. Rowe, and F. Koetter, Collage city, Cambridge, Massachusetts: The MIT Press, 1978.

[16] C. Sitte, City Planning According to Artistic Principles, New York: Random House, 1965. (First published in 1889).

[17] D. Koch, Architecture Re-configured, The Journal of Space Syntax, vol. 1-1, 2010, pp.1-16.

[18] E. Bacon, Design of Cities, New York: Joseph Aronson, 1967.

[19] E. Howard, Garden Cities of To-morrow, Swan Sonnenschein \& Co., Ltd, 1898.

[20] E. Llabres, and E. RICO, Proxi Modelling: A Tacit Approach to Territorial Praxis, The Journal of Space Syntax, vol. 5-1, 2014, pp.5067.

[21] F. Otto, Occupying and Connecting: Thoughts on Territories and Spheres of Influence, with Particular Reference to Human Settlement, Stuttgart: Axel Menges, 2009.

[22] G. Broadbent, Emerging Concepts in Urban Space Design, UK: Taylor \& Francis, 1995.

[23] G. Cullen, The Concise Townscape, New York: Van Nostrand Reinhold Co., 1961.

[24] H. Frichot, On the Becoming-Indiscernible of the Diagram in Societies of Control, The Journal of Space Syntax, vol.5-1, 2014, pp.1-14.

[25] H. Kato, E. Iwasaki, A. El Shazly, and G. Yutaka, Regional Diversity and Residential Development on the Edge of Greater Cairo - Linking Three kinds of Data - Census, Household Survey and Geographical Data - with GIS to Greater Cairo. In: A. Okabe (ed.) GIS-based Studies in the Humanities and Social Sciences, UK: Taylor \& Francis, 2006, pp.191210.

[26] H. Markhede, P. Miranda, and D. Koch, Spatial Positioning Tool: Background, Prototype Software and some Correlation Data, The Journal of Space Syntax, vol. 1-1, 2010, pp.149-163.

[27] H. Schadelbach, Hybrid Spatial Topologies, The Journal of Space Syntax, vol. 3-2, 2012, pp.204-222.

[28] J. Burry, The Construction of a Problem: Architecture Modelling After Descartes, The Journal of Space Syntax, vol. 5-1, 2014, pp.15-34.

[29] J. Hanson, Decoding Homes and Houses, Cambridge: Cambridge University Press, 1998.

[30] J. Jacobs, The Death and Life of Great American Cities, Random House, 1961.

[31] J. Steen, and H. Markhede, Spatial and Social Configurations in Offices, The Journal of Space Syntax, vol.1-1, 2010, pp.121-132.

[32] K. Al-Sayed, How Design Evolve, The Journal of Space Syntax, vol. 51, 2014, pp.68-90.

[33] K. Lynch, Good City Form, Cambridge, Massachusetts: The MIT Press, 1981.

[34] K. Lynch, The Image of the City, Cambridge, Massachusetts: The MIT Press, 1960.

[35] K. Richer, F. Schmid, and P. Laube, Semantic Trajectory Compression: Representing Urban Movement in a Nutshell, Journal of Spatial Information Science, vol. 4, 2012, pp.3-30.

[36] L. Helme, C. Derix, and A. Izaki, A. Spatial Configuration: Semiautomatic Methods for Layout Generation in Practice, The Journal of Space Syntax, vol. 5-1, 2014, pp.35-49.

[37] L. Marcus, Spatial Capital: A Proposal for an Extension of Space Syntax into a More General Urban Morphology, The Journal of Space Syntax, vol. 1-1, 2010, pp.30-40.
[38] L. Mumford, The City in History: Its Origins, its Transformations, and its Prospects, New York: Harcourt Brace Jovanovich, Inc., 1961.

[39] M. Conzen, Thinking About Urban Form: Papers on Urban Morphology, 1932-1998, New York: Peter Lang, 2004.

[40] M. Goodchild, Twenty Years of Progress: GIScience in 2010, Journal of Spatial Information Science, vol.1, 2010, pp.3-20.

[41] M. Kruger, and A. Vieira, Scaling Relative Asymmetry in Space Syntax Analysis, The Journal of Space Syntax, vol. 3-2, 2012, pp.194-203.

[42] M. Ostwald, Examining the Relationship Between Topology and Geometry: A Configurationally Analysis of the Rural Houses (19842005) of Glenn Murcutt, The Journal of Space Syntax, vol. 2-2, 2011, pp.223-246.

[43] M. Serra, and P. Pinho, Tackling the Structure of Very Large Spatial Systems - Space Syntax and the Analysis of Metropolitan Form, The Journal of Space Syntax, vol. 4-2, 2013, pp.179-196.

[44] M. Tafuri, and F. Dal Co, Modern Architecture I, Nrew York: Electa/Rizzoli, 1991

[45] N. Dalton, and R. Dalton, Solutions for Visibility-Accessibility and Signage Problems via Layered-Graphs, The Journal of Space Syntax, vol. 1-1, 2010, pp.164-176.

[46] O. Newman, Creating Defensible Space, Alabama:Diane Pub Co., 1996.

[47] P. Blanchard, and D. Volchenkov, Mathematical Analysis of Urban Spatial Networks, Berlin: Springer, 2009.

[48] P. Geddes, Cities in Evolution, London: Benn Bros. Ltd., 1949.

[49] P. Osmond, The Convex Space as the 'Atom' of Urban Analysis, The Journal of Space Syntax, vol. 2-1, 2011, pp.97-114.

[50] R. Krier, Urban Space, New York:International Publications, 1979.

[51] R. Unwin, Town Planning in Practice, New York: Princeton Architectural Press, Inc., 1994. (First Published in 1909).

[52] S. Chapin, E. Kaiser, and D. Godschalk, Urban Land Use Planning, Urbana: University of Illinois Press, 1965.

[53] S. Hanna, The Inverted Genotype and its Implications for the Flexibility of Architectural Models, The Journal of Space Syntax, vol. 2-2, 2011, pp.247-272.

[54] S. Kostof, The City Shaped: Urban Patterns and Meanings Through History, London: Thames \& Hudson Ltd., 1991.

[55] S. Psarra, The Ghost of Conceived Space: What Kind of Work does or should Space Syntax Perform for Architecture?, The Journal of Space Syntax, vol. 1-1, 2010, pp.17-29.

[56] S. Psarra, and S. Mcelhinney, Just Around the Corner From Where You Are: Probabilistic Isovist Fields, Interface and Embodied Projection, The Journal of Space Syntax, vol. 5-1, 2014, pp.109-132.

[57] S. Winter, and C. Freksa, Approaching the Notion of Place by Contrast, Journal of Spatial Information Science, vol. 5, 2012, 31-50.

[58] T. Varoudis, and S. Psarra, Beyond Two-dimensions: Architecture Through Three-dimensional Visibility Graph Analysis, The Journal of Space Syntax, vol.5-1, 2014, pp.91-108.

[59] W. Luo, and A. Mac Eacheren, A. Geo-social Visual Analytics, Journal of Spatial Information Science, vol. 8, 2014, 27-66.

[60] Y. Ye, and A. Van Nes, Measuring Urban Maturation Process in Dutch and Chinese New Towns: Combining Street Network Configuration with Building Density and Degree of Land Use Diversification Through GIS, The Journal of Space Syntax, vol. 4-1, 2013, pp.18-37.

About Author (s):

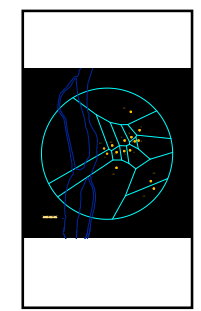

"Apparently, most of research efforts in space syntax are directed towards the bridging from perceptive spatial configuration into the virtual reality of conceptual design at different levels of building resolutions, with little or no attention paid to the driving 'basic design' force of interchangeable convex space." 